\title{
A NEW PROOF OF A UNICITY THEOREM OF MEROMORPHIC FUNCTIONS AND THEIR DERIVATIVES
}

\author{
Jianming Chang \\ Changshu Institute of Technology, Department of Mathematics \\ Changshu, Jiangsu 215500, P. R. China; jmchang@cslg.edu.cn
}

\begin{abstract}
We give a new proof of the following result by a uniform method: If a nonconstant meromorphic function $f$ shares two distinct finite values CM with its derivative $f^{(k)}$, then $f$ is an entire function that satisfies $f^{(k)} \equiv f$.
\end{abstract}

\section{Introduction}

Let $f$ and $g$ be two functions meromorphic on the plane $\mathbf{C}$ and $a$ a complex value. Then it is said that $f$ and $g$ share the value $a$ CM if $f(z)-a$ and $g(z)-a(1 / f(z)$ and $1 / g(z)$ for $a=\infty)$ have the same zeros, counting multiplicity. If the multiplicities are ignored, then we say $f$ and $g$ share the value $a$ IM. See [15]. Using his well-known theory, Nevanlinna [13] proved in 1929 the Five-Value Theorem: If two nonconstant meromorphic functions $f$ and $g$ share five values IM, then $f \equiv g$. In 1976, Rubel and Yang [14] studied the unicity relation between an entire function and its derivative and proved that if a nonconstant entire function $f$ and its derivative $f^{\prime}$ share two values $a, b \mathrm{CM}$, then $f^{\prime} \equiv f$. Later on, many interesting results $[1-5,7,10-12]$ on this subject have been done. The following well-known theorem is one representative result.

Theorem 1. Let $k \geq 1$ be an integer. If a nonconstant meromorphic function $f$ and its derivative $f^{(k)}$ share two finite values CM, then $f$ is an entire function that satisfies $f^{(k)} \equiv f$.

This result for the case $k=1$ was proved by Gundersen [7] and Mues and Steinmetz [12] independently, while for the case of higher-order derivatives, it is due to Frank and Ohlenroth [4] for the case that the shared values are nonzero and Frank and Weissenborn [5] for the case that one of the shared values is zero.

We remark that these proofs for Theorem 1 are all based on Nevanlinna's theory but the methods are different for different cases. For the case of higher-order derivatives, besides Nevanlinna's two fundamental theorems and the lemma of logarithmic derivatives, the proofs require essentially some deep results in Nevanlinna's theory. The papers $[4,5]$ used a result of Frank and Weissenborn [6] on estimating the number of poles by the number of zeros of derivatives while the paper [11] relied

doi:10.5186/aasfm.2012.3702

2010 Mathematics Subject Classification: Primary 30D35.

Key words: Entire functions, meromorphic functions, shared values, unicity theorem.

Research supported by the NNSF of China (Grant Nos. 10871094 and 11171045), SRF for ROCS, SEM, NSFU of Jiangsu, China (Grant No. 08KJB110001) and the Qing Lan Project of Jiangsu, China. 
on a result of Hayman and Miles [9] on the growth relation between a transcendental function and its derivatives.

In this paper, we give a new proof of the above well-known result by a uniform method. In our proof, combined with some careful calculation on the coefficients of Laurent series, only the Nevanlinna's first fundamental theorem and the lemma of logarithmic derivatives are used. The auxiliary functions we construct are also convoluted, but the way of construction seems more natural. Our method can also be used to treat the case of sharing small functions.

Throughout this paper, we use the standard notions of Nevanlinna's theory $[8,16]$ such as $T(r, f), m(r, f), N(r, f)$, etc. In particular, The notion $S(r, f)$ is defined to be any function in $r$ that satisfies $S(r, f)=o(T(r, f))$ as $r \rightarrow \infty$, possibly outside a set of $r$ of finite linear measure. A function $a$ is said to be small with respect to $f$ if $T(r, a)=S(r, f)$.

\section{Auxiliary results}

The preliminary results that we require are only the well-known Nevanlinna's first fundamental theorem, the lemma of logarithmic derivatives $[8,16]$ with a simple fact.

Nevanlinna's first fundamental theorem (NFT1). Let $f$ be a nonconstant meromorphic function on the plane $\mathbf{C}$ and $a \in \mathbf{C}$ a constant. Then

$$
T\left(r, \frac{1}{f-a}\right)=T(r, f)+O(1)
$$

Lemma of logarithmic derivatives (LLD). Let $f$ be a nonconstant meromorphic function on the plane $\mathbf{C}$. Then for every positive integer $k$

$$
m\left(r, \frac{f^{(k)}}{f}\right)=S(r, f) .
$$

Lemma 3. Let $A, B, C, D$ be nonzero constants. If each zero of $e^{A z}-B$ is a zero of $e^{C z}-D$, then there exists a nonzero integer $k$ such that $C=k A$.

Proof. Let $z_{1}$ be a zero of $e^{A z}-B$. Then $z_{2}=z_{1}+\frac{2 \pi}{A} i$ is also a zero of $e^{A z}-B$. Thus by the condition, $e^{C\left(z_{2}-z_{1}\right)}=1$. It follows that $C\left(z_{2}-z_{1}\right)=2 k \pi i$ for some $k \in \mathbf{Z} \backslash\{0\}$. And so $C=k A$.

\section{Proof of Theorem 1}

In this section, we give the new proof of Theorem 1. It contains two main steps. In the first step, we prove that $f$ have few poles, while in the second step, we finish the proof. In the sequel, we say the two distinct shared values are $a$ and $b$.

Step 1. We prove that $N(r, f)=S(r, f)$ by reduction to absurdity. So we assume henceforth in this step that

$$
N(r, f) \neq S(r, f) .
$$

Since the shared values $a$ and $b$ are distinct, we can assume that $|a| \leq|b|$, so that $b \neq 0$. 
By (1), $f$ has poles in C. Since $f$ and $f^{(k)}$ share the values $a$ and $b \mathrm{CM}$, the following two auxiliary functions

$$
h:=\frac{f-a}{f^{(k)}-a} \quad \text { and } \quad g:=\frac{f-b}{f^{(k)}-b}
$$

take on nonzero finite values at the zeros of $f^{(k)}-a$ (or $f-a$ ) and the zeros of $f^{(k)}-b$ (or $f-b$ ), respectively. Thus $h$ and $g$ are entire functions and have the same zeros which are just the poles of $f$ and each of which has exact multiplicity $k$. It follows that $h / g$ has no zeros and poles and thus there exists an entire function $\alpha$ such that

$$
\begin{aligned}
e^{\alpha} & =\frac{h}{g}=\frac{(f-a)\left(f^{(k)}-b\right)}{\left(f^{(k)}-a\right)(f-b)} \\
& =1+(b-a)\left(\frac{1}{f-b}-\frac{1}{f^{(k)}-a}\right)-\frac{(b-a)^{2}}{(f-b)\left(f^{(k)}-a\right)} .
\end{aligned}
$$

We claim that $e^{\alpha}$, and hence $\alpha$ can not be constant. Indeed, if $e^{\alpha}$ is constant, then $e^{\alpha}=1$ since the value of the right side of (3) at a pole of $f$ is 1 . Thus by (3), $h \equiv g$ and hence $f^{(k)} \equiv f$, which contradicts the fact that $f$ has poles. Thus $e^{\alpha}$ and hence $\alpha$ is nonconstant. By NFT1 and LLD, it follows easily from (3) that $T\left(r, e^{\alpha}\right)=O(T(r, f))$ and hence $T\left(r, \alpha^{\prime}\right)=m\left(r, \alpha^{\prime}\right)=m\left(r,\left(e^{\alpha}\right)^{\prime} / e^{\alpha}\right)=S(r, f)$. Further, by (3), it is not difficult to see that each pole of $f$ with multiplicity $p \geq 2$ is a zero of $e^{\alpha}-1$ with multiplicity $p$ and hence a zero of $\alpha^{\prime}$ with multiplicity $p-1 \geq \frac{1}{2} p$. Thus $N_{(2}(r, f) \leq 2 N\left(r, 1 / \alpha^{\prime}\right)=S(r, f)$, where $N_{(2}(r, f)$ is the counting function of multiple poles of $f$, counting multiplicity. Thus by (1), the counting function $N_{1}(r, f)$ of simple poles of $f$ satisfies

$$
N_{1}(r, f)=N(r, f)-N_{(2}(r, f) \neq S(r, f)
$$

Since $g$ is an entire function and all zeros of $g$ have the same multiplicity $k$, there exists an entire function $G$, all of whose zeros are simple, such that

$$
g=G^{k}
$$

Next, before we give the detailed proof, we show the outline of our proof. First by computing out $\frac{G^{\prime \prime}}{G^{\prime}}, \frac{G^{\prime \prime \prime}}{G^{\prime}}$ (and $\frac{G^{(4)}}{G^{\prime}}$ for $\left.k=1,2\right)$ at simple poles of $f$ using the Laurent series, we obtain some small functions $\phi_{k}, \psi_{k}$ (and $\omega_{k}$ for $\left.k=1,2\right)$, see (23), (54)-(55) and (72) below. Then by eliminating the higher-order derivatives $G^{\prime \prime}, G^{\prime \prime \prime}$ and $G^{(4)}$, we obtain some relations like $A G^{\prime}=B G$ with $A$ and $B$ being small, see (25), (57) and (61) below. Some analysis then shows that the small functions $A$ and $B$ must be vanishing, and then by some further analysis, we arrive at some contradictions.

Now we continue the proof. Let $z_{0}$ be a simple pole of $f$. Then $e^{\alpha\left(z_{0}\right)}=1$ and $\alpha^{\prime}\left(z_{0}\right) \neq 0$. Near $z_{0}, f$ has the following expansion

$$
f(z)=\frac{a_{0}}{z-z_{0}}+a_{1}+a_{2}\left(z-z_{0}\right)+a_{3}\left(z-z_{0}\right)^{2}+O\left[\left(z-z_{0}\right)^{3}\right] \quad\left(a_{0} \neq 0\right),
$$


where $a_{i}$ are constants. Thus

$$
\begin{aligned}
f^{\prime} & =-\frac{a_{0}}{\left(z-z_{0}\right)^{2}}+a_{2}+2 a_{3}\left(z-z_{0}\right)+O\left[\left(z-z_{0}\right)^{2}\right], \\
f^{\prime \prime} & =\frac{2 a_{0}}{\left(z-z_{0}\right)^{3}}+2 a_{3}+O\left(z-z_{0}\right), \\
f^{(j)} & =\frac{(-1)^{j} j ! a_{0}}{\left(z-z_{0}\right)^{j+1}}+O(1)=\frac{(-1)^{j} j ! a_{0}}{\left(z-z_{0}\right)^{j+1}}\left(1+O\left[\left(z-z_{0}\right)^{j+1}\right]\right) .
\end{aligned}
$$

By a computation, the function $e^{\alpha}$ can be expanded as

$$
\begin{aligned}
e^{\alpha}= & 1+\alpha^{\prime}\left(z_{0}\right)\left(z-z_{0}\right)+\frac{1}{2} \alpha^{\prime}\left(z_{0}\right) \beta\left(z_{0}\right)\left(z-z_{0}\right)^{2} \\
& +\frac{1}{6} \alpha^{\prime}\left(z_{0}\right)\left[\left(\beta\left(z_{0}\right)\right)^{2}+\beta^{\prime}\left(z_{0}\right)\right]\left(z-z_{0}\right)^{3} \\
& +\frac{1}{24} \alpha^{\prime}\left(z_{0}\right)\left[\left(\beta\left(z_{0}\right)\right)^{3}+3 \beta\left(z_{0}\right) \beta^{\prime}\left(z_{0}\right)+\beta^{\prime \prime}\left(z_{0}\right)\right]\left(z-z_{0}\right)^{4} \\
& +O\left[\left(z-z_{0}\right)^{4}\right]
\end{aligned}
$$

where

$$
\beta:=\alpha^{\prime}+\frac{\alpha^{\prime \prime}}{\alpha^{\prime}}
$$

Next we consider three cases.

Case 1. We assume $k \geq 3$. Then by (3) with (6) and (9)-(10), near $z_{0}$

$$
\begin{aligned}
\frac{1}{f-b} & =\frac{e^{\alpha}-1}{b-a}+\frac{1}{f(k)-a}+\frac{b-a}{(f-b)\left(f^{(k)}-a\right)} \\
& =\frac{e^{\alpha}-1}{b-a}+\frac{\left(z-z_{0}\right)^{k+1}}{(-1)^{k} k ! a_{0}}+O\left[\left(z-z_{0}\right)^{k+2}\right] \\
& =\left(\frac{e^{\alpha}-1}{b-a}+\frac{\left(z-z_{0}\right)^{k+1}}{(-1)^{k} k ! a_{0}}\right)\left(1+\frac{O\left[\left(z-z_{0}\right)^{k+2}\right]}{\frac{e^{\alpha}-1}{b-a}+\frac{\left(z-z_{0}\right)^{k+1}}{(-1)^{k} k ! a_{0}}}\right) \\
& =\left(\frac{e^{\alpha}-1}{b-a}+\frac{\left(z-z_{0}\right)^{k+1}}{(-1)^{k} k ! a_{0}}\right)\left(1+O\left[\left(z-z_{0}\right)^{k+1}\right]\right) .
\end{aligned}
$$

Thus $\left(\frac{1}{f-b}\right)_{z_{0}}^{\prime}=\frac{\alpha^{\prime}\left(z_{0}\right)}{b-a}$. However, by $(6)$, we have $\left(\frac{1}{f-b}\right)_{z_{0}}^{\prime}=\frac{1}{a_{0}}$. It follows that $a_{0}=\frac{b-a}{\alpha^{\prime}\left(z_{0}\right)}$. Hence by (9) with $j=k,(10)$ and (12), with the notion

$$
c=\frac{(-1)^{k} k ! a_{0}}{b-a}=\frac{(-1)^{k} k !}{\alpha^{\prime}\left(z_{0}\right)}
$$


we get that

$$
\begin{aligned}
G^{k}= & g=\frac{f-b}{f^{(k)}-b}=\frac{\frac{\left(z-z_{0}\right)^{k+1}}{(-1)^{k} k ! a_{0}}}{\frac{e^{\alpha}-1}{b-a}+\frac{\left(z-z_{0}\right)^{k+1}}{(-1)^{k} k ! a_{0}}}\left(1+O\left[\left(z-z_{0}\right)^{k+1}\right]\right) \\
= & \frac{\left(z-z_{0}\right)^{k+1}}{c\left(e^{\alpha}-1\right)+\left(z-z_{0}\right)^{k+1}}\left(1+O\left[\left(z-z_{0}\right)^{k+1}\right]\right) \\
= & \frac{\left(z-z_{0}\right)^{k}}{(-1)^{k} k !}\left[1-\frac{1}{2} \beta\left(z_{0}\right)\left(z-z_{0}\right)\right. \\
& \left.\quad+\frac{1}{12}\left(\left[\beta\left(z_{0}\right)\right]^{2}-2 \beta^{\prime}\left(z_{0}\right)\right)\left(z-z_{0}\right)^{2}+O\left[\left(z-z_{0}\right)^{3}\right]\right] .
\end{aligned}
$$

On the other hand, we have

$$
\begin{aligned}
& G^{k}=\left[G^{\prime}\left(z_{0}\right)+\right.\left.\frac{1}{2} G^{\prime \prime}\left(z_{0}\right)\left(z-z_{0}\right)+\frac{1}{6} G^{\prime \prime \prime}\left(z_{0}\right)\left(z-z_{0}\right)^{2}+O\left[\left(z-z_{0}\right)^{3}\right]\right]^{k}\left(z-z_{0}\right)^{k} \\
&=\left[G^{\prime}\left(z_{0}\right)\right]^{k}\left\{1+\frac{k}{2} G_{2}\left(z-z_{0}\right)\right. \\
&\left.+\left[\frac{k}{6} G_{3}+\frac{k(k-1)}{8}\left(G_{2}\right)^{2}\right]\left(z-z_{0}\right)^{2}+O\left[\left(z-z_{0}\right)^{3}\right]\right\}\left(z-z_{0}\right)^{k}
\end{aligned}
$$

where

$$
G_{j}=\frac{G^{(j)}\left(z_{0}\right)}{G^{\prime}\left(z_{0}\right)}, \quad j=2,3 .
$$

Now comparing the coefficients of (15) and (16) yields that

$$
\begin{aligned}
{\left[G^{\prime}\left(z_{0}\right)\right]^{k} } & =\frac{1}{(-1)^{k} k !}, \\
\frac{k}{2} \frac{G^{\prime \prime}\left(z_{0}\right)}{G^{\prime}\left(z_{0}\right)} & =-\frac{1}{2} \beta\left(z_{0}\right), \\
\frac{k}{6} \frac{G^{\prime \prime \prime}\left(z_{0}\right)}{G^{\prime}\left(z_{0}\right)}+\frac{k(k-1)}{8}\left(\frac{G^{\prime \prime}\left(z_{0}\right)}{G^{\prime}\left(z_{0}\right)}\right)^{2} & =\frac{1}{12}\left(\left[\beta\left(z_{0}\right)\right]^{2}-2 \beta^{\prime}\left(z_{0}\right)\right) .
\end{aligned}
$$

By (19) and (20), we have

$$
\begin{aligned}
G^{\prime \prime}\left(z_{0}\right)+\frac{1}{k} \beta\left(z_{0}\right) G^{\prime}\left(z_{0}\right) & =0, \\
G^{\prime \prime \prime}\left(z_{0}\right)+\left(\frac{k-3}{4 k^{2}}\left[\beta\left(z_{0}\right)\right]^{2}+\frac{1}{k} \beta^{\prime}\left(z_{0}\right)\right) G^{\prime}\left(z_{0}\right) & =0 .
\end{aligned}
$$

These computations show that the possible poles of the functions

$$
\phi_{k}:=\frac{G^{\prime \prime}+\frac{1}{k} \beta G^{\prime}}{G} \text { and } \psi_{k}:=\frac{G^{\prime \prime \prime}+\left(\frac{k-3}{4 k^{2}} \beta^{2}+\frac{1}{k} \beta^{\prime}\right) G^{\prime}}{G}
$$

come from the multiple poles of $f$, the poles of $\beta$ and the poles of $\frac{k-3}{4 k^{2}} \beta^{2}+\frac{1}{k} \beta^{\prime}$, and hence come from the zeros of $\alpha^{\prime}$. Thus we have $N\left(r, \phi_{k}\right)+N\left(r, \psi_{k}\right)=S(r, f)$. Further, by LLD, we know that $m\left(r, \phi_{k}\right)+m\left(r, \psi_{k}\right)=S(r, f)$, so that

$$
T\left(r, \phi_{k}\right)+T\left(r, \psi_{k}\right)=S(r, f) \text {. }
$$


By the definition of the function $\phi_{k}$ in (23), we have $G^{\prime \prime}=-\frac{1}{k} \beta G^{\prime}+\phi_{k} G$, and hence $G^{\prime \prime \prime}=-\frac{1}{k} \beta G^{\prime \prime}-\frac{1}{k} \beta^{\prime} G^{\prime}+\phi_{k}^{\prime} G+\phi_{k} G^{\prime}=\left(\phi_{k}-\frac{1}{k} \beta^{\prime}+\frac{1}{k^{2}} \beta^{2}\right) G^{\prime}+\left(\phi_{k}^{\prime}-\frac{1}{k} \beta \phi_{k}\right) G$. It with the definition of the function $\psi_{k}$ in (23) yields that

$$
\left(\phi_{k}+\frac{k+1}{4 k^{2}} \beta^{2}\right) G^{\prime}=\left(\psi_{k}+\frac{1}{k} \beta \phi_{k}-\phi_{k}^{\prime}\right) G \text {. }
$$

If $\phi_{k}+\frac{k+1}{4 k^{2}} \beta^{2} \not \equiv 0$, then by (25), (24), NFT1 and the fact that $G$ is an entire function with only simple zeros, we get

$$
\begin{aligned}
N_{1}(r, f) & \leq N\left(r, \frac{1}{G}\right)=N\left(r, \frac{\psi_{k}+\frac{1}{k} \beta \phi_{k}-\phi_{k}^{\prime}}{\phi_{k}+\frac{k+1}{4 k^{2}} \beta^{2}}\right) \\
& \leq N\left(r, \psi_{k}+\frac{1}{k} \beta \phi_{k}-\phi_{k}^{\prime}\right)+T\left(r, \phi_{k}+\frac{k+1}{4 k^{2}} \beta^{2}\right)+O(1)=S(r, f) .
\end{aligned}
$$

This contradicts (8). Thus $\phi_{k}+\frac{k+1}{4 k^{2}} \beta^{2} \equiv 0$, and hence

$$
\phi_{k}=-\frac{k+1}{4 k^{2}} \beta^{2} \text {. }
$$

Now let

$$
\gamma:=\frac{g^{\prime}}{g}=k \frac{G^{\prime}}{G}
$$

Then $\frac{G^{\prime}}{G}=\frac{1}{k} \gamma$ and $\frac{G^{\prime \prime}}{G}=\frac{1}{k} \gamma^{\prime}+\frac{1}{k^{2}} \gamma^{2}$. Thus by (27) and the definition of the function $\phi_{k}$ in (23), we get

$$
\gamma^{\prime}+\frac{1}{k} \gamma^{2}+\frac{1}{k} \beta \gamma+\frac{k+1}{4 k} \beta^{2}=0 .
$$

Next we show that (29) is impossible. Suppose that $z_{0}$ is a simple pole of $f$. Let

$$
H:=\frac{c \alpha^{\prime} e^{\alpha}}{c\left(e^{\alpha}-1\right)+\left(z-z_{0}\right)^{k+1}} .
$$

Then by the facts that $c\left(e^{\alpha}-1\right)+\left(z-z_{0}\right)^{k+1}=(-1)^{k} k !\left(z-z_{0}\right)+O\left[\left(z-z_{0}\right)^{2}\right]$ and that $c \alpha^{\prime} e^{\alpha}=(-1)^{k} k !+O\left(z-z_{0}\right)$,

$$
\begin{aligned}
H^{\prime} & =\frac{\left.c\left[\left(\alpha^{\prime}\right)^{2}+\alpha^{\prime \prime}\right)\right] e^{\alpha}}{c\left(e^{\alpha}-1\right)+\left(z-z_{0}\right)^{k+1}}-\frac{c \alpha^{\prime} e^{\alpha}\left[c \alpha^{\prime} e^{\alpha}+(k+1)\left(z-z_{0}\right)^{k}\right]}{\left[c\left(e^{\alpha}-1\right)+\left(z-z_{0}\right)^{k+1}\right]^{2}} \\
& =\beta H-H^{2}-\frac{k+1}{(-1)^{k} k !}\left(z-z_{0}\right)^{k-2}+O\left[\left(z-z_{0}\right)^{k-1}\right] .
\end{aligned}
$$

Thus by (14), near $z_{0}$

$$
\begin{aligned}
\gamma & =\frac{g^{\prime}}{g}=\frac{k+1}{z-z_{0}}-\frac{c \alpha^{\prime} e^{\alpha}+(k+1)\left(z-z_{0}\right)^{k}}{c\left(e^{\alpha}-1\right)+\left(z-z_{0}\right)^{k+1}}+O\left[\left(z-z_{0}\right)^{k}\right] \\
& =\frac{k+1}{z-z_{0}}-H-\frac{k+1}{(-1)^{k} k !}\left(z-z_{0}\right)^{k-1}+O\left[\left(z-z_{0}\right)^{k}\right] \\
& =\frac{k+1}{z-z_{0}}-H+O\left[\left(z-z_{0}\right)^{k-1}\right] .
\end{aligned}
$$


By (30), we have

$$
\begin{aligned}
\gamma^{2}= & \frac{(k+1)^{2}}{\left(z-z_{0}\right)^{2}}+H^{2}-2(k+1) \frac{H}{z-z_{0}}-\frac{2(k+1)^{2}}{(-1)^{k} k !}\left(z-z_{0}\right)^{k-2} \\
& +\frac{2(k+1)}{(-1)^{k} k !}\left(z-z_{0}\right)^{k-1} H+O\left[\left(z-z_{0}\right)^{k-1}\right] \\
= & \frac{(k+1)^{2}}{\left(z-z_{0}\right)^{2}}+H^{2}-2(k+1) \frac{H}{z-z_{0}}-\frac{2(k+1) k}{(-1)^{k} k !}\left(z-z_{0}\right)^{k-2}+O\left[\left(z-z_{0}\right)^{k-1}\right], \\
\gamma^{\prime}= & -\frac{k+1}{\left(z-z_{0}\right)^{2}}-H^{\prime}-\frac{(k+1)(k-1)}{(-1)^{k} k !}\left(z-z_{0}\right)^{k-2}+O\left[\left(z-z_{0}\right)^{k-1}\right] \\
= & -\frac{k+1}{\left(z-z_{0}\right)^{2}}-\beta H+H^{2}-\frac{(k+1)(k-2)}{(-1)^{k} k !}\left(z-z_{0}\right)^{k-2}+O\left[\left(z-z_{0}\right)^{k-1}\right] .
\end{aligned}
$$

Substituting (31)-(33) into (29), we can get

$$
\left(H-\frac{1}{z-z_{0}}-\frac{1}{2} \beta\right)^{2}=\frac{k^{2}}{(-1)^{k} k !}\left(z-z_{0}\right)^{k-2}+O\left[\left(z-z_{0}\right)^{k-1}\right] .
$$

Since near $z_{0}$,

$$
H-\frac{1}{z-z_{0}}-\frac{1}{2} \beta=\frac{1}{12}\left[\left(\beta\left(z_{0}\right)\right)^{2}-2 \beta^{\prime}\left(z_{0}\right)\right]\left(z-z_{0}\right)+O\left[\left(z-z_{0}\right)^{2}\right],
$$

by (34) and (35), we see that $k \geq 4$, and for $k>4,\left(\beta\left(z_{0}\right)\right)^{2}-2 \beta^{\prime}\left(z_{0}\right)=0$ while for $k=4,\left[\left(\beta\left(z_{0}\right)\right)^{2}-2 \beta^{\prime}\left(z_{0}\right)\right]^{2}=96$. Since $N_{1}(r, f) \neq S(r, f)$ and $T(r, \beta)=S(r, f)$, it follows that $\beta^{2}-2 \beta^{\prime} \equiv 0$ for $k>4$ and $\left(\beta^{2}-2 \beta^{\prime}\right)^{2} \equiv 96$ for $k=4$.

For the former case, by $\beta^{2}-2 \beta^{\prime} \equiv 0$, we get

$$
\beta=-\frac{2}{z+c}
$$

for some constant $c$. Since $\beta=\alpha^{\prime}+\frac{\alpha^{\prime \prime}}{\alpha^{\prime}}=\frac{\left(\alpha^{\prime} e^{\alpha}\right)^{\prime}}{\alpha^{\prime} e^{\alpha}}$, we get

$$
\alpha^{\prime} e^{\alpha}=\frac{d}{(z+c)^{2}}
$$

for some constant $d$. This is impossible.

Thus $k=4$ and $\left(\beta^{2}-2 \beta^{\prime}\right)^{2} \equiv 96$. A similar argument shows that $\alpha^{\prime}$ and $\beta$ are constants satisfying $\left(\alpha^{\prime}\right)^{4}=\beta^{4}=96$. Thus by (23) and (27), $G$ satisfies the equation $G^{\prime \prime}+\frac{1}{4} \beta G^{\prime}+\frac{5}{64} \beta^{2} G=0$. Solving this equation yields that

$$
G(z)=C_{1} e^{-\frac{1-2 i}{8} \beta z}+C_{2} e^{-\frac{1+2 i}{8} \beta z}=e^{-\frac{1+2 i}{8} \beta z}\left(C_{1} e^{\frac{1}{2} i \beta z}+C_{2}\right),
$$

where $C_{1}$ and $C_{2}$ are constants. Since $\alpha^{\prime}$ is a nonzero constant, $f$ has no multiple poles, so that $N(r, 1 / G)=N_{1}(r, f) \neq S(r, f)$. Thus the constants $C_{1}$ and $C_{2}$ are nonzero. We claim that

$$
e^{\frac{1}{2} i \beta z}=-\frac{C_{2}}{C_{1}} \Longrightarrow e^{\frac{1}{2} \beta z}=D
$$


for some constant $D$. In fact, let $z_{1}$ be a zero of $e^{\frac{1}{2} i \beta z}+\frac{C_{2}}{C_{1}}$, then by $(36), G\left(z_{1}\right)=0$ and hence $z_{1}$ is a simple pole of $f$, so that by $(18),\left[G^{\prime}\left(z_{1}\right)\right]^{4}=\frac{1}{24}$. By $(36)$, we have

$$
\begin{aligned}
{\left[G^{\prime}(z)\right]^{4} } & =\left(\frac{1-2 i}{8} C_{1} \beta e^{-\frac{1-2 i}{8} \beta z}+\frac{1+2 i}{8} C_{2} \beta e^{-\frac{1+2 i}{8} \beta z}\right)^{4} \\
& =\beta^{4} e^{-\frac{1}{2} \beta z}\left(e^{-\frac{1}{2} i \beta z}\right)^{2}\left(\frac{1-2 i}{8} C_{1} \beta e^{\frac{1}{2} i \beta z}+\frac{1+2 i}{8} C_{2}\right)^{4}
\end{aligned}
$$

By letting $z=z_{1}$ in (38), we see that

$$
e^{\frac{1}{2} \beta z_{1}}=24 \beta^{4}\left(C_{1} C_{2}\right)^{2}\left(-\frac{1-2 i}{8} \beta+\frac{1+2 i}{8}\right)^{4} .
$$

This proves the claim (37). However, by Lemma 3, (37) is impossible.

Case 2. We now assume that $k=2$. The process of the proof is similar to that of Case 1. Near the simple pole $z_{0}$ of $f$, by (3) with (6), (8) and (10), we have

$$
\begin{aligned}
\frac{1}{f-b}= & \frac{e^{\alpha}-1}{b-a}+\frac{1}{f^{\prime \prime}-a}+\frac{b-a}{(f-b)\left(f^{\prime \prime}-a\right)} \\
= & \frac{\alpha^{\prime}\left(z_{0}\right)}{b-a}\left(z-z_{0}\right)+\frac{\alpha^{\prime}\left(z_{0}\right) \beta\left(z_{0}\right)}{2(b-a)}\left(z-z_{0}\right)^{2} \\
& +\left(\frac{\alpha^{\prime}\left(z_{0}\right)}{6(b-a)}\left[\left(\beta\left(z_{0}\right)\right)^{2}+\beta^{\prime}\left(z_{0}\right)\right]+\frac{1}{2 a_{0}}\right)\left(z-z_{0}\right)^{3}+O\left[\left(z-z_{0}\right)^{4}\right] .
\end{aligned}
$$

However, by (6), we have

$$
\begin{aligned}
\frac{1}{f-b}= & \frac{z-z_{0}}{a_{0}+\left(a_{1}-b\right)\left(z-z_{0}\right)+a_{2}\left(z-z_{0}\right)^{2}+O\left[\left(z-z_{0}\right)^{3}\right]} \\
= & \frac{1}{a_{0}}\left(z-z_{0}\right)-\frac{a_{1}-b}{\left(a_{0}\right)^{2}}\left(z-z_{0}\right)^{2}-\left(\frac{a_{2}}{\left(a_{0}\right)^{2}}-\frac{\left(a_{1}-b\right)^{2}}{\left(a_{0}\right)^{3}}\right)\left(z-z_{0}\right)^{3} \\
& +O\left[\left(z-z_{0}\right)^{4}\right] .
\end{aligned}
$$

Thus by (40) and (41), we see that

$$
\begin{aligned}
\frac{1}{a_{0}} & =\frac{\alpha^{\prime}\left(z_{0}\right)}{b-a}, \\
\frac{a_{1}-b}{\left(a_{0}\right)^{2}} & =-\frac{\alpha^{\prime}\left(z_{0}\right) \beta\left(z_{0}\right)}{2(b-a)}, \\
\frac{a_{2}}{\left(a_{0}\right)^{2}}-\frac{\left(a_{1}-b\right)^{2}}{\left(a_{0}\right)^{3}} & =-\frac{\alpha^{\prime}\left(z_{0}\right)}{6(b-a)}\left[\left(\beta\left(z_{0}\right)\right)^{2}+\beta^{\prime}\left(z_{0}\right)\right]-\frac{1}{2 a_{0}} .
\end{aligned}
$$

It follows from (42)-(44) that

$$
a_{0}=\frac{b-a}{\alpha^{\prime}\left(z_{0}\right)}, a_{1}=b-\frac{1}{2} a_{0} \beta\left(z_{0}\right), a_{2}=\frac{a_{0}}{12}\left(\left[\beta\left(z_{0}\right)\right]^{2}-2 \beta^{\prime}\left(z_{0}\right)-6\right) .
$$


Thus by (6), (8) and (45),

$$
\begin{aligned}
& G^{2}=g=\frac{f-b}{f^{\prime \prime}-b} \\
& =\frac{a_{0}+\left(a_{1}-b\right)\left(z-z_{0}\right)+a_{2}\left(z-z_{0}\right)^{2}+a_{3}\left(z-z_{0}\right)^{3}+O\left[\left(z-z_{0}\right)^{4}\right]}{2 a_{0}+\left(2 a_{3}-b\right)\left(z-z_{0}\right)^{3}+O\left[\left(z-z_{0}\right)^{4}\right]}\left(z-z_{0}\right)^{2} \\
& \text { (46) }=\frac{1}{2}\left[1+\frac{a_{1}-b}{a_{0}}\left(z-z_{0}\right)+\frac{a_{2}}{a_{0}}\left(z-z_{0}\right)^{2}+\frac{b}{2 a_{0}}\left(z-z_{0}\right)^{3}\right. \\
& \left.+O\left[\left(z-z_{0}\right)^{4}\right]\right]\left(z-z_{0}\right)^{2} \\
& =\frac{1}{2}\left[1-\frac{1}{2} \beta\left(z_{0}\right)\left(z-z_{0}\right)+\frac{1}{12}\left(\left[\beta\left(z_{0}\right)\right]^{2}-2 \beta^{\prime}\left(z_{0}\right)-6\right)\left(z-z_{0}\right)^{2}\right. \\
& \left.+\frac{b \alpha^{\prime}\left(z_{0}\right)}{2(b-a)}\left(z-z_{0}\right)^{3}+O\left[\left(z-z_{0}\right)^{4}\right]\right]\left(z-z_{0}\right)^{2} .
\end{aligned}
$$

On the other hand, we have

$$
\begin{aligned}
G^{2}=[ & G^{\prime}\left(z_{0}\right)+\frac{1}{2} G^{\prime \prime}\left(z_{0}\right)\left(z-z_{0}\right)+\frac{1}{6} G^{\prime \prime \prime}\left(z_{0}\right)\left(z-z_{0}\right)^{2} \\
& \left.+\frac{1}{24} G^{(4)}\left(z_{0}\right)\left(z-z_{0}\right)^{3}+O\left[\left(z-z_{0}\right)^{4}\right]\right]^{2}\left(z-z_{0}\right)^{2} \\
=[ & \left.G^{\prime}\left(z_{0}\right)\right]^{2}\left[1+\frac{G^{\prime \prime}\left(z_{0}\right)}{G^{\prime}\left(z_{0}\right)}\left(z-z_{0}\right)+\left(\frac{G^{\prime \prime \prime}\left(z_{0}\right)}{3 G^{\prime}\left(z_{0}\right)}+\frac{\left[G^{\prime \prime}\left(z_{0}\right)\right]^{2}}{4\left[G^{\prime}\left(z_{0}\right)\right]^{2}}\right)\left(z-z_{0}\right)^{2}\right. \\
& \left.+\left(\frac{G^{(4)}\left(z_{0}\right)}{12 G^{\prime}\left(z_{0}\right)}+\frac{G^{\prime \prime}\left(z_{0}\right) G^{\prime \prime \prime}\left(z_{0}\right)}{6\left[G^{\prime}\left(z_{0}\right)\right]^{2}}\right)\left(z-z_{0}\right)^{3}+O\left[\left(z-z_{0}\right)^{4}\right]\right]\left(z-z_{0}\right)^{2} .
\end{aligned}
$$

By comparing the coefficients of (46) and (47), we get

$$
\begin{aligned}
{\left[G^{\prime}\left(z_{0}\right)\right]^{2} } & =\frac{1}{2}, \\
\frac{G^{\prime \prime}\left(z_{0}\right)}{G^{\prime}\left(z_{0}\right)} & =-\frac{1}{2} \beta\left(z_{0}\right), \\
\frac{G^{\prime \prime \prime}\left(z_{0}\right)}{3 G^{\prime}\left(z_{0}\right)}+\frac{\left[G^{\prime \prime}\left(z_{0}\right)\right]^{2}}{4\left[G^{\prime}\left(z_{0}\right)\right]^{2}} & =\frac{1}{12}\left(\left[\beta\left(z_{0}\right)\right]^{2}-2 \beta^{\prime}\left(z_{0}\right)-6\right), \\
\frac{G^{(4)}\left(z_{0}\right)}{12 G^{\prime}\left(z_{0}\right)}+\frac{G^{\prime \prime}\left(z_{0}\right) G^{\prime \prime \prime}\left(z_{0}\right)}{6\left[G^{\prime}\left(z_{0}\right)\right]^{2}} & =\frac{b \alpha^{\prime}\left(z_{0}\right)}{2(b-a)} .
\end{aligned}
$$

It follows that

$$
\begin{array}{r}
G^{\prime \prime}+\left.\frac{1}{2} \beta G^{\prime}\right|_{z_{0}}=0, \\
G^{\prime \prime \prime}-\left.\frac{1}{16}\left(\beta^{2}-8 \beta^{\prime}-24\right) G^{\prime}\right|_{z_{0}}=0, \\
G^{(4)}-\left.\frac{1}{16}\left(\beta^{3}-8 \beta \beta^{\prime}-24 \beta^{\prime}+\frac{96 b}{b-a} \alpha^{\prime}\right) G^{\prime}\right|_{z_{0}}=0 .
\end{array}
$$


Thus by the same argument used to prove (27), it follows from (51)-(53) that the functions

$$
\begin{aligned}
& \phi_{2}:=\frac{G^{\prime \prime}+\frac{1}{2} \beta G^{\prime}}{G}, \quad \psi_{2}:=\frac{G^{\prime \prime \prime}-\frac{1}{16}\left(\beta^{2}-8 \beta^{\prime}-24\right) G^{\prime}}{G}, \\
& \omega_{2}:=\frac{G^{(4)}-\frac{1}{16}\left(\beta^{3}-8 \beta \beta^{\prime}-24 \beta^{\prime}+\frac{96 b}{b-a} \alpha^{\prime}\right) G^{\prime}}{G}
\end{aligned}
$$

satisfy

$$
T\left(r, \phi_{2}\right)+T\left(r, \psi_{2}\right)+T\left(r, \omega_{2}\right)=S(r, f) .
$$

By the definition of $\phi_{2}, G^{\prime \prime}=-\frac{1}{2} \beta G^{\prime}+\phi_{2} G$, and then $G^{\prime \prime \prime}=\left(\frac{1}{4} \beta^{2}-\frac{1}{2} \beta^{\prime}+\phi_{2}\right) G^{\prime}+$ $\left(-\frac{1}{2} \beta \phi_{2}+\phi_{2}^{\prime}\right) G$. This with the definition of $\psi_{2}$ shows that

$$
\left(\frac{3}{16} \beta^{2}+\frac{3}{2}+\phi_{2}\right) G^{\prime}=\left(\psi_{2}+\frac{1}{2} \beta \phi_{2}-\phi_{2}^{\prime}\right) G \text {. }
$$

If $\frac{3}{16} \beta^{2}+\frac{3}{2}+\phi_{2} \not \equiv 0$, then as in Case 1 , we have by (57)

$$
\begin{aligned}
N_{1}(r, f) & \leq N\left(r, \frac{1}{G}\right) \leq N\left(r, \psi_{2}+\frac{1}{2} \beta \phi_{2}-\phi_{2}^{\prime}\right)+N\left(r, \frac{1}{\frac{3}{16} \beta^{2}+\frac{3}{2}+\phi_{2}}\right) \\
& \leq T\left(r, \psi_{2}+\frac{1}{2} \beta \phi_{2}-\phi_{2}^{\prime}\right)+T\left(r, \frac{3}{16} \beta^{2}+\frac{3}{2}+\phi_{2}\right)+O(1) \\
& =S(r, f) .
\end{aligned}
$$

This contradicts (4). Thus $\frac{3}{16} \beta^{2}+\frac{3}{2}+\phi_{2} \equiv 0$, and hence

$$
\phi_{2}=-\frac{3}{16} \beta^{2}-\frac{3}{2}
$$

so that by the definition of $\phi_{2}, G^{\prime \prime}=-\frac{1}{2} \beta G^{\prime}-\left(\frac{3}{16} \beta^{2}+\frac{3}{2}\right) G$, and hence by differentiating two times,

$$
\begin{aligned}
G^{(4)}= & \frac{1}{16}\left(\beta^{3}+24 \beta-8 \beta^{\prime \prime}\right) G^{\prime} \\
& +\left(-\frac{3}{256} \beta^{4}+\frac{3}{8} \beta^{2} \beta^{\prime}+\frac{3}{16} \beta^{2}-\frac{3}{8}\left(\beta^{\prime}\right)^{2}-\frac{3}{8} \beta \beta^{\prime \prime}+\frac{3}{2} \beta^{\prime}+\frac{9}{4}\right) G .
\end{aligned}
$$

Thus by (55) and (60),

$$
\left(\beta \beta^{\prime}+3 \beta+3 \beta^{\prime}-\beta^{\prime \prime}-\frac{12 b}{b-a} \alpha^{\prime}\right) G^{\prime}=2\left(w_{2}-A\right) G,
$$

where $A$ is the coefficient of $G$ in (60). Using (4) again and (61), we have

$$
\beta \beta^{\prime}+3 \beta+3 \beta^{\prime}-\beta^{\prime \prime}-\frac{12 b}{b-a} \alpha^{\prime}=0 .
$$

Since $\beta=\alpha^{\prime}+\frac{\alpha^{\prime \prime}}{\alpha^{\prime}}$, by (62), we see that $\alpha^{\prime}$ has no zeros, so that $\alpha^{\prime}=e^{\tau}$ for some entire function $\tau$. Thus by (62), we have

$$
\tau^{\prime} e^{2 \tau}+\left(3 \tau^{\prime}+3-\frac{12 b}{b-a}\right) e^{\tau}+\tau^{\prime} \tau^{\prime \prime}+3 \tau^{\prime}+3 \tau^{\prime \prime}-\tau^{\prime \prime \prime}=0
$$

It follows that $\tau$ is a constant and hence $a=-3 b$. Since $a \neq b$ and we have assumed $|a| \leq|b|$, this is impossible. 
Case 3. We now consider the final case $k=1$. In this case, $G=g$. The process of the proof is similar to that of Case 2. Near the simple pole $z_{0}$ of $f$, we have

$$
\begin{aligned}
\frac{1}{f-b}= & \frac{e^{\alpha}-1}{b-a}+\frac{1}{f^{\prime}-a}+\frac{b-a}{(f-b)\left(f^{\prime}-a\right)} \\
= & \frac{\alpha^{\prime}\left(z_{0}\right)}{b-a}\left(z-z_{0}\right)+\left(\frac{\alpha^{\prime}\left(z_{0}\right) \beta\left(z_{0}\right)}{2(b-a)}-\frac{1}{a_{0}}\right)\left(z-z_{0}\right)^{2} \\
& +\left(\frac{\alpha^{\prime}\left(z_{0}\right)}{6(b-a)}\left[\left(\beta\left(z_{0}\right)\right)^{2}+\beta^{\prime}\left(z_{0}\right)\right]-\frac{b-a}{\left(a_{0}\right)^{2}}\right)\left(z-z_{0}\right)^{3} \\
& +\left(\frac{\alpha^{\prime}\left(z_{0}\right)}{24(b-a)}\left[\left(\beta\left(z_{0}\right)\right)^{3}+3 \beta\left(z_{0}\right) \beta^{\prime}\left(z_{0}\right)+\beta^{\prime \prime}\left(z_{0}\right)\right]\right. \\
& \left.\quad+\frac{(b-a)\left(a_{1}-b\right)}{\left(a_{0}\right)^{3}}-\frac{a_{2}-a}{\left(a_{0}\right)^{2}}\right)\left(z-z_{0}\right)^{4}+O\left[\left(z-z_{0}\right)^{5}\right] .
\end{aligned}
$$

However, by (6), we have

$$
\begin{aligned}
\frac{1}{f-b} & =\frac{z-z_{0}}{a_{0}+\left(a_{1}-b\right)\left(z-z_{0}\right)+a_{2}\left(z-z_{0}\right)^{2}+O\left[\left(z-z_{0}\right)^{3}\right]} \\
& =\frac{1}{a_{0}}\left(z-z_{0}\right)-\frac{a_{1}-b}{\left(a_{0}\right)^{2}}\left(z-z_{0}\right)^{2}-\left(\frac{a_{2}}{\left(a_{0}\right)^{2}}-\frac{\left(a_{1}-b\right)^{2}}{\left(a_{0}\right)^{3}}\right)\left(z-z_{0}\right)^{3} \\
& -\left(\frac{a_{3}}{\left(a_{0}\right)^{2}}-\frac{2\left(a_{1}-b\right) a_{2}}{\left(a_{0}\right)^{3}}+\frac{\left(a_{1}-b\right)^{3}}{\left(a_{0}\right)^{4}}\right)\left(z-z_{0}\right)^{4}+O\left[\left(z-z_{0}\right)^{5}\right] .
\end{aligned}
$$

Thus as dong in Case 2, by comparing the coefficients of (64) and (65), we can compute out the coefficients $a_{0}, a_{1}, a_{2}$ and $a_{3}$ in proper order:

$$
\begin{aligned}
& a_{0}=\left.\frac{b-a}{\alpha^{\prime}}\right|_{z_{0}}, \quad a_{1}=b-\left.\frac{1}{2}(\beta-2) a_{0}\right|_{z_{0}}, \\
& a_{2}=\left.\left(\frac{1}{12} \beta^{2}-\beta-\frac{1}{6} \beta^{\prime}+1+\alpha^{\prime}\right)\right|_{z_{0}} a_{0} \\
& a_{3}=\left.\left[\frac{1}{2} \beta^{2}+\frac{1}{24} \beta \beta^{\prime}-\frac{5}{2} \beta-\frac{1}{2} \beta^{\prime}-\frac{1}{24} \beta^{\prime \prime}+2-\left(\frac{\beta}{2}-\frac{2 b-3 a}{b-a}\right) \alpha^{\prime}\right]\right|_{z_{0}} a_{0} .
\end{aligned}
$$

Thus by (6), (7) and (66),

$$
\begin{aligned}
& g=\frac{f-b}{f^{\prime}-b} \\
& =\frac{a_{0}+\left(a_{1}-b\right)\left(z-z_{0}\right)+a_{2}\left(z-z_{0}\right)^{2}+a_{3}\left(z-z_{0}\right)^{3}+O\left[\left(z-z_{0}\right)^{4}\right]}{-a_{0}+\left(a_{2}-b\right)\left(z-z_{0}\right)^{2}+2 a_{3}\left(z-z_{0}\right)^{3}+O\left[\left(z-z_{0}\right)^{4}\right]}\left(z-z_{0}\right) \\
& =-\left[1+\frac{a_{1}-b}{a_{0}}\left(z-z_{0}\right)+\frac{2 a_{2}-b}{a_{0}}\left(z-z_{0}\right)^{2}\right. \\
& \left.+\left(\frac{3 a_{3}}{a_{0}}+\frac{\left(a_{1}-b\right)\left(a_{2}-b\right)}{\left(a_{0}\right)^{2}}\right)\left(z-z_{0}\right)^{3}++O\left[\left(z-z_{0}\right)^{4}\right]\right]\left(z-z_{0}\right) \\
& =-\left(z-z_{0}\right)\left[1+X\left(z_{0}\right)\left(z-z_{0}\right)\right. \\
& \left.+Y\left(z_{0}\right)\left(z-z_{0}\right)^{2}+Z\left(z_{0}\right)\left(z-z_{0}\right)^{3}+O\left[\left(z-z_{0}\right)^{4}\right]\right],
\end{aligned}
$$


where

$$
\begin{aligned}
X:= & -\frac{1}{2}(\beta-2), \\
Y:= & \frac{1}{6} \beta^{2}-2 \beta-\frac{1}{3} \beta^{\prime}+2+\frac{b-2 a}{b-a} \alpha^{\prime}, \\
Z:= & -\frac{1}{24} \beta^{3}+\frac{25}{12} \beta^{2}+\frac{5}{24} \beta \beta^{\prime}-9 \beta-\frac{5}{3} \beta^{\prime}-\frac{1}{8} \beta^{\prime \prime}+7 \\
& -\left(\frac{3 b-4 a}{2 b-2 a} \beta-\frac{2 b-4 a}{b-a}\right) \alpha^{\prime} .
\end{aligned}
$$

By (67), we see that

$$
g^{\prime}\left(z_{0}\right)=-1, g^{\prime \prime}\left(z_{0}\right)=-2 X\left(z_{0}\right), g^{\prime \prime \prime}\left(z_{0}\right)=-6 Y\left(z_{0}\right), g^{(4)}\left(z_{0}\right)=-24 Z\left(z_{0}\right) .
$$

As in Case 2, it follows from (71) and LLD that the functions

$$
\phi_{1}:=\frac{g^{\prime \prime}-2 X g^{\prime}}{g}, \quad \psi_{1}:=\frac{g^{\prime \prime \prime}-6 Y g^{\prime}}{g}, \quad \omega_{1}:=\frac{g^{(4)}-24 Z g^{\prime}}{g}
$$

satisfy

$$
T\left(r, \phi_{1}\right)+T\left(r, \psi_{1}\right)+T\left(r, \omega_{1}\right)=S(r, f) .
$$

By the definition of $\phi_{1}$, we have $g^{\prime \prime}=2 X g^{\prime}+\phi_{1} g$ so that $g^{\prime \prime \prime}=\left(2 X^{\prime}+4 X^{2}+\phi_{1}\right) g^{\prime}+$ $\left(2 X \phi_{1}+\phi_{1}^{\prime}\right) g$. This with the definition of $\psi_{1}$ shows that $\left(2 X^{\prime}+4 X^{2}-6 Y+\phi_{1}\right) g^{\prime}=$ $\left(\psi_{1}-2 X \phi_{1}-\phi_{1}^{\prime}\right) g$. Then as showed above, it follows from (4) and (73) that

$$
\phi_{1}=-2 X^{\prime}-4 X^{2}+6 Y, \quad \psi_{1}=2 X \phi_{1}+\phi_{1}^{\prime} .
$$

And by $g^{\prime \prime \prime}=6 Y g^{\prime}+\psi_{1} g$, we have $g^{(4)}=\left(6 Y^{\prime}+12 X Y+\psi_{1}\right) g^{\prime}+\left(6 \phi_{1} Y+\psi_{1}^{\prime}\right) g$. This with $g^{(4)}=24 Z g^{\prime}+\omega_{1} g$ shows that $\left(6 Y^{\prime}+12 X Y-24 Z+\psi_{1}\right) g^{\prime}=\left(\omega_{1}-6 \phi_{1} Y-\psi_{1}^{\prime}\right) g$. As above, it follows from (4) and (73) that $6 Y^{\prime}+12 X Y-24 Z+\psi_{1}=0$. It with (74) shows that

$$
12 Y^{\prime}+24 X Y-12 X X^{\prime}-8 X^{3}-2 X^{\prime \prime}-24 Z=0 .
$$

Substituting (68)-(70) into (75), we get

$$
-28 \beta^{2}+156 \beta+14 \beta^{\prime}-104+24 \alpha^{\prime} \beta-\frac{24 b-48 a}{b-a} \alpha^{\prime}+\frac{12 b-24 a}{b-a} \alpha^{\prime \prime}=0 .
$$

Since $\beta=\alpha^{\prime}+\frac{\alpha^{\prime \prime}}{\alpha^{\prime}}$, by (76), we see that $\alpha^{\prime}$ has no zeros so that $\alpha^{\prime}=e^{\tau}$ for some entire function $\tau$. Thus $\beta=e^{\tau}+\tau^{\prime}$. Therefore, it is not difficult to see from (76) that $\tau$ and hence $\alpha^{\prime}=\beta$ are constants satisfying

$$
\beta^{2}-\frac{33 b-27 a}{b-a} \beta+26=0
$$

Since $\alpha^{\prime}=\beta$ are constant, by (68) and (69), we have $X=-\frac{1}{2}(\beta-2)$ and $Y=$ $\frac{1}{6} \beta^{2}-\frac{b}{b-a} \beta+2$. And thus by (74), $\phi_{1}=-\frac{2 b+4 a}{b-a} \beta+8$. Therefore, by (72), $g$ satisfies the differential equation

$$
g^{\prime \prime}+(\beta-2) g^{\prime}+\left(\frac{2 b+4 a}{b-a} \beta-8\right) g=0 .
$$

Since $N(r, 1 / g)=N_{1}(r, f) \neq S(r, f)$, the characteristic equation of (78)

$$
\lambda^{2}+(\beta-2) \lambda+\frac{2 b+4 a}{b-a} \beta-8=0
$$


has two distinct roots $\lambda_{1}(\neq 0)$ and $\lambda_{2}$, and $g$ has the form

$$
g=C_{1} e^{\lambda_{1} z}+C_{2} e^{\lambda_{2} z},
$$

where $C_{1}$ and $C_{2}$ are nonzero constants. By $(71), g(z)=0 \Longrightarrow g^{\prime}(z)=-1$. Thus by (80), we see that

$$
e^{\left(\lambda_{1}-\lambda_{2}\right) z}=-\frac{C_{2}}{C_{1}} \Longrightarrow e^{\lambda_{2} z}=C_{3},
$$

where $C_{3}$ is a constant. It now follows from Lemma 3 that there exists an integer $n$ such that

$$
\lambda_{2}=n\left(\lambda_{1}-\lambda_{2}\right) .
$$

For convenience, set

$$
\mu=\frac{b}{a-b} .
$$

Since $\lambda_{1}$ and $\lambda_{2}$ are the roots of (79),

$$
\begin{aligned}
\lambda_{1}+\lambda_{2} & =-\beta+2, \\
\lambda_{1} \lambda_{2} & =(6 \mu-4) \beta-8 .
\end{aligned}
$$

By (82) and (84), we get $\lambda_{1} \lambda_{2}=\frac{m}{4 m+1}(\beta-2)^{2}$, where $m=n(n+1) \geq 0$. Thus by (83) and (85), we get

$$
\begin{aligned}
\beta^{2}-(6 \mu+27) \beta+26 & =0, \\
\frac{m}{4 m+1}(\beta-2)^{2}-(6 \mu-4) \beta+8 & =0 .
\end{aligned}
$$

Eliminating $\mu$ by (86) and (87), we get

$$
\beta^{2}-\frac{120 m+31}{3 m+1} \beta+\frac{68 m+18}{3 m+1}=0 .
$$

Thus

$$
\beta=\beta_{ \pm}=\frac{120 m+31 \pm \sqrt{13584 m^{2}+6952 m+889}}{2(3 m+1)}
$$

is a positive real number. It can be verified that $\beta_{+}>26$ and $0<\beta_{-}<1$. Hence either $\beta>26$ or $0<\beta<1$. By (78), we have

$$
6 \mu=\frac{\beta^{2}+26}{\beta}-27=\frac{(\beta-1)(\beta-26)}{\beta} .
$$

So $\mu$ is a positive real number. Thus by (83) and $b \neq 0,|a|=\frac{\mu+1}{\mu}|b|>|b|$, which contradicts the assumption that $|a| \leq|b|$.

Step 2. We now finish the proof by assuming that $f^{(k)} \not \equiv f$. By Step 1 , we have now

$$
N(r, f)=S(r, f) .
$$

Since $f$ and $f^{(k)}$ share $a$ and $b$ CM, we have obviously $f^{(k)}-a \not \equiv 0$ and $f^{(k)}-b \not \equiv 0$. We claim that $h$ and $g$ defined in (2) are nonconstant.

In fact, if $h$ is constant, then $h \neq 0$ since $f$ is nonconstant. Also by $f^{(k)} \not \equiv f$, $h \neq 1$. If $f$ takes the value $b$ at some point $z_{0}$, then by the condition, $f^{(k)}\left(z_{0}\right)=b$. Thus by (2), we get $h=1$, which is ruled out. So $f \neq b$, and hence $f^{(k)} \neq b$. By (2) 
and the assumption that $h$ is constant, $f$ satisfies the linear differential equation of constant coefficients

$$
h f^{(k)}-f+(1-h) a=0 .
$$

By solving the linear differential equation (92), we see that $f$ is a linear combination of finitely many exponential functions $e^{\lambda z}, \lambda \in \mathbf{C}$ with constant or polynomial coefficients. Thus $f$ is an entire function of order at most one, so that by $f \neq b$, $f=b+C e^{A z}$ for some nonzero constants $A$ and $C$. Thus $f^{(k)}=C A^{k} e^{A z}$. Since $f^{(k)} \neq b$, we get $b=0$ and hence $a \neq 0$. Thus $f=C e^{A z}$. Substituting it into (92) gives that $C\left(h A^{k}-1\right) e^{A z}+(1-h) a=0$. It follows that $C\left(h A^{k}-1\right)=(1-h) a=0$. Since $a \neq 0$, we get $h=1$. However, this is ruled out. The claim is proved.

By (2) and (91), we have

$$
N(r, 1 / h)+N(r, 1 / g) \leq 2 k N(r, f)=S(r, f) .
$$

Case 1. Assume that $a=0$. Then by LLD, we get $m(r, 1 / h)=S(r, f)$. Thus by $(93), T(r, 1 / h)=m(r, 1 / h)+N(r, 1 / h)=S(r, f)$, and hence by NFT1, $T(r, h)=$ $T(r, 1 / h)+O(1)=S(r, f)$, so that by LLD,

$$
\begin{aligned}
m\left(r, \frac{1}{f-b}\right) & =m\left(r, \frac{b f^{(k)}}{f(f-b)} \cdot \frac{h}{b}\right) \leq m\left(r, \frac{b f^{(k)}}{f(f-b)}\right)+m\left(r, \frac{h}{b}\right) \\
& =m\left(r, \frac{f^{(k)}}{f-b}-\frac{f^{(k)}}{f}\right)+S(r, f)=S(r, f) .
\end{aligned}
$$

Further, since $f$ and $f^{(k)}$ share the nonzero value $b$ CM, each zero of $f(z)-b$ of multiplicity $p$ must be a zero of $h-1$ of multiplicity at least $p$. Thus by NFT1 and the fact that $T(r, h)=S(r, f)$, we have

$$
N\left(r, \frac{1}{f-b}\right) \leq N\left(r, \frac{1}{h-1}\right) \leq T(r, h)+O(1)=S(r, f) .
$$

It now follows from (94), (95) and NFT1,

$$
T(r, f)=m\left(r, \frac{1}{f-b}\right)+N\left(r, \frac{1}{f-b}\right)+O(1)=S(r, f) .
$$

This is a contradiction.

Case 2. Now suppose that $a \neq 0$. Then by (2), we have

$$
\frac{h^{\prime}}{h}=\frac{f^{\prime}}{f-a}-\frac{f^{(k+1)}}{f^{(k)}-a} .
$$

It follows that

$$
\begin{aligned}
\frac{1}{f-b} & =\frac{h}{h^{\prime}}\left[\frac{f^{\prime}}{(f-a)(f-b)}-\frac{f^{(k+1)}}{\left(f^{(k)}-a\right) f^{(k)}} \cdot \frac{f^{(k)}}{f-b}\right] \\
& =\frac{h}{h^{\prime}}\left[\frac{1}{a-b}\left(\frac{f^{\prime}}{f-a}-\frac{f^{\prime}}{f-b}\right)-\frac{f^{(k)}}{a(f-b)}\left(\frac{f^{(k+1)}}{f^{(k)}-a}-\frac{f^{(k+1)}}{f^{(k)}}\right)\right] .
\end{aligned}
$$


Thus by LLD and NFT1, we get

$$
\begin{aligned}
m\left(r, \frac{1}{f-b}\right) & \leq m\left(r, \frac{h}{h^{\prime}}\right)+S(r, f) \leq T\left(r, \frac{h^{\prime}}{h}\right)+S(r, f) \\
& \leq N\left(r, \frac{h^{\prime}}{h}\right)+S(r, f) \leq \bar{N}(r, f)+S(r, f)=S(r, f) .
\end{aligned}
$$

Similarly, by considering the function $g$, we also have

$$
m\left(r, \frac{1}{f-a}\right)=S(r, f) \text {. }
$$

Further, since $f$ and $f^{(k)}$ share the nonzero values $a, b \mathrm{CM}$ and $f^{(k)} \not \equiv f$, we have by LLD, NFT1 and (91)

$$
\begin{aligned}
N\left(r, \frac{1}{f-a}\right)+N\left(r, \frac{1}{f-b}\right) & \leq N\left(r, \frac{1}{\frac{f^{(k)}}{f}-1}\right) \leq T\left(r, \frac{f^{(k)}}{f}-1\right)+O(1) \\
& \leq N\left(r, f^{(k)} / f\right)+m\left(r, f^{(k)} / f\right)+O(1) \\
& \leq k \bar{N}(r, f)+N(r, 1 / f)+S(r, f) \\
& \leq T(r, f)+S(r, f) .
\end{aligned}
$$

By (99)-(101) and NFT1, we now have

$$
\begin{aligned}
2 T(r, f)= & T\left(r, \frac{1}{f-a}\right)+T\left(r, \frac{1}{f-b}\right)+O(1) \\
= & m\left(r, \frac{1}{f-a}\right)+m\left(r, \frac{1}{f-b}\right) \\
& +N\left(r, \frac{1}{f-a}\right)+N\left(r, \frac{1}{f-b}\right)+O(1) \\
\leq & T(r, f)+S(r, f) .
\end{aligned}
$$

This is also impossible. The proof of Theorem 1 is thus complete.

Acknowledgement. I am grateful to the referee for his valuable suggestions.

\section{References}

[1] Chang, J. M., and M. L. FAng: On entire functions that share a value with their derivatives. - Ann. Acad. Sci. Fenn. Math. 31, 2006, 265-286.

[2] Chang, J. M., and M. L. FAng: Normal families and uniqueness of entire functions and their derivatives. - Acta Math. Sinica 23, 2007, 973-986.

[3] Chang, J. M., M. L. FAng, and L. Zalcman: Entire functions that share a set with their derivatives. - Arch. Math. 89, 2007, 561-569.

[4] Frank, G., and W. Ohlenroth: Meromorphe Funktionen, die mit einer ihrer Ableitungen Werte teilen. - Complex Variables 6, 1986, 23-37 .

[5] Frank, G., and G. Weissenborn: Meromorphe Funktionen, die mit einer ihrer Ableitungen Werte teilen. - Complex Variables 7, 1986, 33-43.

[6] Frank, G., and G. Weissenborn: Rational deficient functions of meromorphic functions. Bull. London Math. Soc. 18, 1986, 29-33.

[7] Gundersen, G. G.: Meromorphic functions that share two finite values with their derivatives. - Pacific J. Math. 105, 1983, 299-309. 
[8] Hayman, W. K.: Meromorphic functions. - Clarendon Press, Oxford, 1964.

[9] Hayman, W. K., and J. Miles: On the growth of a meromorphic function and its derivatives. - Complex Variables 12, 1989, 245-260.

[10] Jank, G., E. Mues, and L. Volkmann: Meromorphe Funktionen, die mit ihrer ersten und zweiten Ableitung einen endlichen Wert teilen. - Complex Variables 6, 1986, 51-71.

[11] LI, P.: Unicity of meromorphic functions and their derivatives. - J. Math. Anal. Appl. 285, 2003, 651-665.

[12] Mues, E., and N. Steinmetz: Meromorphe funktionen, die mit ihrer Ableitung Werte teilen. - Manuscripta Math. 29, 1979, 195-206.

[13] Nevanlinna, R.: Le théorème de Picard-Borel et la théorie des fonctions méromorphes. Gauthier-Villars, Paris, 1929.

[14] Rubel, L. A., and C. C. Yang: Values shared by an entire function and its derivative. - In: Complex Analysis, Kentucky 1976 (Proc. Conf.), Lecture Notes in Math. 599, Springer-Verlag, Berlin, Heidelberg, New York, 1977, 101-103.

[15] YANG, C. C., and H. X. Yı: Uniqueness theory of meromorphic functions. - Science Press, Beijing, 1995 (in Chinese); Kluwer Academic Publishers, The Netherlands, 2003 (English transl.).

[16] YANG, L.: Value distribution theory. Springer-Verlag, Berlin, 1993.

Received 13 April 2010 